- Assessment and care planning including hydration and nutrition

- End of life care

- Care after death/bereavement care

On completion the participant receives a passport at a celebration event, and will have compiled a personal portfolio of group work, additional tasks, and personal reflections. The organisation receives an annual certificate detailing the number of staff that complete the PCEP, and a portfolio of evidence. We also encourage participants to access e-ELCA and record this on the passport.

Results Evaluation shows encouraging signs of increased motivation, confidence and skills of those attending, and changes being made within their organisations.

It is currently funded via the hospice and MPET (multi professional education \& training), and due to the high uptake, the CCG (clinical commissioning group) have requested further modules to be developed for disease specific training.

Conclusion The PCEP accredits the care worker, and inspires them to make changes from within their organisation.

\section{P-245 INTRODUCING MULTI-DISCIPLINARY REFLECTIVE PRACTICE GROUPS INTO CHILDREN'S PALLIATIVE CARE SERVICES}

Victoria Montgomery, Linda Maynard, Carolyn Leese, Lorraine Kaka, Josie Dwyer. East Anglia's Children's Hospices (EACH), Milton, UK

\subsection{6/bmjspcare-2016-001245.266}

Background Effective clinical supervision benefits patient care by:

- Enabling staff to challenge practice in a confidential environment and reflect on feedback separate from managerial considerations

- Enhancing staff wellbeing through enabling exploration and management of both the personal and professional demands created by the nature of their work in palliative care.

Aims To introduce multi-disciplinary reflective practice groups (MRPGs) into three children's hospice services.

Method Ninety-minutes sessions followed a structured nine-step process, led by an experienced facilitator. Groups had open membership, ran with 4-10 participants and there was mandatory minimum attendance. Group membership included care assistants, nurses, counsellors, art and music therapists and play specialists but excluded care managers. Participants brought a clinical work related dilemma to share, with one dilemma being chosen and discussed throughout the session.

Post every session, participants rated the reflection and identified learning by giving an example of "One thing I am going to take away from today's session is..."

Results Between November 2015 and April 2016, 31 MRPGs took place, with 156 participants, totalling 106 different staff.

$90 \%$ of responses agreed or strongly agreed that MRPGs were useful, with positive response rate increasing over the six month period.

In eight out of 31 groups, $>75 \%$ of participants strongly agreed that MRPGs were useful. All eight of these groups discussed a clinical nursing related dilemma rather than a psychosocial focussed issue. Key themes in analysis included 'normalising and validating'; 'improving practice/skills in work with families'; 'reflection' and 'taking action'.

Conclusions Contrary to staff expectations MRPGs were perceived useful by staff themselves. Key learning areas reported were a good fit with background reasons for introducing the sessions. Our next steps are to:

- Collect examples of perceived impact on the quality of clinical work with children and families across the different disciplines

- Widen participation to include bank staff, volunteers and hospice employees not providing direct clinical work.

\section{P-246 'WE'RE BOLD AND CURIOUS!' - AN EVALUATION OF LEADING FROM THE MIDDLE PROGRAMME, TWO YEARS ON...}

Liz Arnold, Nigel Hartley. Earl Mountbatten Hospice, Newport, UK

\subsection{6/bmjspcare-2016-001245.267}

Help the Hospices Commission into the Future of Hospice Care (2012) recommended that hospices need to ensure their workforce is fit for purpose and that good leadership is not undermined by a 'missing middle tier' of management. In response, a local bespoke leadership development programme was developed, entitled, 'Leading from the Middle' (LfM), using the 'Future Ambitions for Hospice Care' (2013) report as a strategic framework to ground the learning. Since 2014, forty members of staff from across the hospice have participated in the programme.

The work of cohort one was pioneering and had an immediate impact on organisational culture. This achievement was further consolidated by cohort two who realised the 'day to day' practicality of 'leadership at all levels' and the challenges for staff. The two cohorts have delivered a comprehensive picture of what a 'Well-Led' hospice looks like, through a balance of strategic and operational perspectives. The evaluation report revealed specific and tangible examples of leadership development:

- The consistency of the hospice vision gave 'permission' for staff to take responsibility and the hospice values enabled people to be 'bold' and 'curious'

- Collaborative working across the hospice which has resulted in innovative responses to operational challenges

- Practical demonstrations of service development

- Tangible evidence of capability and confidence in strategic thinking and analysis

- Mid-level managers and professionals communicated their views and challenged the status

- Concrete evidence of the potential and value of working with diversity

- Improved ability to challenge, contribute, innovate and use feedback

- Practical examples of learning applied to issues and challenges

- Greater motivation and shared commitment to responding to challenges.

The community of LfM represents a cornerstone for a 'WellLed' hospice culture where values are fully embraced and the vision of service is secured for the future. 


\section{P-247 FROM MANAGING TO LEADING - UNLOCKING THE HIDDEN TALENT}

Robert Standfield. St. Richard's Hopsice, Worcester, UK

10.1136/bmjspcare-2016-001245.268

One of the key operating principles from the working paper of the Commission into the Future of Hospice Care (2012) identified leadership as a core requirement both at the strategic and operational level within organisations. St. Richard's Hospice has been fortunate in that two members of the senior management team have completed the MA in Hospice Leadership at Lancaster University. We recognised that to embrace the new era of empowering the wider teams and individuals to recognise leadership as a core skill and understand how they can influence current and future development of the sector is core to sustainability.

Up until recently most of the investment in leadership and management has been directed toward individuals where there was an element of self-selection linked to career aspirations or performance management issues where new skills and knowledge were required to deliver their role.

The hospice has now developed and implemented a process where the identification of leadership and management is a bottom-up approach starting at the annual appraisal linked to identifying and nurturing future leaders. We recognised that many of our current staff did not recognise that they already possess inherent skills especially in terms of emotional intelligence, innovation and personal motivation.

As a hospice we have:

- Provided people with the knowledge about how to make best use of appraisal and demonstrating the impact in organisational development.

- Emphasised the hospice strategic objectives, linking their current role to how they are delivering against these objectives and empowering them to contribute to our future strategic direction.

- Establishing longer term career aspirations and navigating people toward achieving these.

- Creating opportunities for staff to complete formal leadership and management qualifications (ILM Levels 3 and 5).

The next stage in the process, identified by the future leaders is establishing the need for coaching and mentoring and how this will support continuous improvement.

\section{P-248 HARMONISING THE WORKING STRUCTURE OF AN IN- PATIENT UNIT EMBEDDING A CULTURE SHIFT}

Jacquie Pamphilon, Karen Causton. St Helena Hospice, Colchester, UK

\subsection{6/bmjspcare-2016-001245.269}

Introduction Hospice nurse leaders need to promote a safe effective environment for patients requiring end-of-life care, whilst being a mentor for less experienced palliative nurses. The hospice inpatient unit manager observed a disparity in roles when registered nurses work as shift leaders. Some shift leaders have not had leadership skills training and do not always have the skills to create process and offer clear direction. Nurse leaders need to achieve good outcomes for all not just a few, to achieve this they need to reflect on and question leadership and management practices (Ellis and Bach 2015)
Aim and objectives

- Coaching RNs to understand leadership practices as shift leaders and the importance of their role in influencing the development of new nurses

- Encourage a culture change for shift leaders to realise the potential of being stong leaders focusing on patient wellbeing.

Approach used - work in progress The unit manager and learning and development officer working in partnership on a joint project investigated reasons for the disparity. Shift leaders spent time reflecting on their approach to leadership, outcomes provided a base of their then situation. Observation allowed for deeper understanding of the nurse's practice (Keatinge 2002). Discussion of the observations included; could understanding and implementing leadership styles harmonise the team, reflection on management theories such as Belbin's team roles (Belbin 2004)

Evaluation RNs recognising their responsibilities as leaders and seeing a growth in project work and knowledge sharing that will educate new nurses.

Conclusion This culture shift is important to bridge gaps in leadership as effective leadership is critical in delivering high quality care and ensuring patient safety and facilitating positive staff development (Frankel 2008). Sharing an approach that looks at leadership styles and what they mean on IPU might offer alternative methods for solutions in other hospices.

\section{P-249 THE ROLE OF HOSPICES IN THE EMERGING WORLD OF INTEGRATED AND DEVOLVED HEALTH AND CARE SYSTEMS}

1,2,3,4,5Steve Dewar, 1,4,5,6,7,8 Judy Taylor, 1,7,8John Harries, 1,5,6,7 Janet Wilson, ${ }_{1,4,7,8}$ Amanda Wilsher. ${ }^{1}$ The Westcott Group Ltd; ${ }^{2}$ Greenwich and Bexley Community Hospice; ${ }^{3}$ St Christopher's Hospice; ${ }^{4}$ Together for Short Lives; ${ }^{5}$ Hospice UK; ${ }^{6}$ NHS Leadership Academy; ${ }^{7}$ Leadership Development, King's Fund; ${ }^{8}$ University of Birmingham

\subsection{6/bmjspcare-2016-001245.270}

Background Strengthening the leadership of hospice care is one of five key steps identified by the Commission into the Future of Hospice Care to ensure that hospices are fit for the future.

The landscape for hospice leaders is complex and challenging. The majority of hospices have seen their statutory funding remain static or even fall. The demand for end-of-life care is predicted to increase significantly and NHS and local authorities are urging local leaders to develop end of life care systems that are integrated, accessible, systematic and responsive.

To meet these challenges, hospice leaders need a coherent story about the role of their organisations in the future, their capacity to contribute to new systems of care and their ability to lead complex change.

Hospice leaders on the Hospice UK Strategic Leadership Programme will be identifying key features of this future landscape for hospice care. The 2016 Conference provides an exciting opportunity to share their emerging vision for the future with the wider hospice community.

Aims Hospice leaders on the Strategic Leadership Programme will be exploring the national landscape of end-of-life care and shaping their own response to the challenges. The aim is to bring these responses to the challenges to the 2016 conference, to stimulate debate and inspire leadership across the sector.

Methods During the first two modules of the Strategic Leadership Programme a cohort of over 30 hospice leaders from more than 20 hospices will develop an understanding of the potential and 